\title{
A new transient ultraluminous X-ray source in NGC 7090
}

\author{
D. J. Walton ${ }^{\circledR},{ }^{1 \star}$ M. Heida ${ }^{\circledR},{ }^{2}$ M. Bachetti ${ }^{\circledR},{ }^{3}$ F. Fürst, ${ }^{4}$ M. Brightman, ${ }^{5}$ H. Earnshaw ${ }^{\circledR}, 5$ P. A. Evans ${ }^{\circledR},{ }^{6}$ \\ A. C. Fabian ${ }^{\oplus}, 1$ B. W. Grefenstette, ${ }^{5}$ F. A. Harrison, ${ }^{5}$ G. L. Israel, ${ }^{7}$ G. B. Lansbury ${ }^{\oplus},{ }^{2}$ M. J. Middleton, ${ }^{8}$ \\ S. Pike, ${ }^{5}$ V. Rana, ${ }^{9}$ T. P. Roberts, ${ }^{10}$ G. A. Rodriguez Castillo, ${ }^{7}$ R. Salvaterra, ${ }^{11}$ X. Song ${ }^{\oplus 12}$ and D. Stern ${ }^{13}$ \\ ${ }^{1}$ Institute of Astronomy, University of Cambridge, Madingley Road, Cambridge CB3 OHA, UK \\ ${ }^{2}$ European Southern Observatory, Karl-Schwarzschild-Straße 2, D-85748 Garching bei München, Germany \\ ${ }^{3}$ INAF - Osservatorio Astronomico di Cagliari, via della Scienza 5, I-09047 Selargius, Italy \\ ${ }^{4}$ Quasar Science Resources for the European Space Agency (ESA), European Space Astronomy Centre (ESAC), Camino Bajo del Castillo s/n, E-28692 \\ Villanueva de la Caada, Madrid, Spain \\ ${ }^{5}$ Cahill Center for Astronomy and Astrophysics, California Institute of Technology, Pasadena, CA 91125, USA \\ ${ }^{6}$ School of Physics, Astronomy, University of Leicester, University Road, Leicester LE1 7RH, UK \\ ${ }^{7}$ INAF - Osservatorio Astronomico di Roma, via Frascati 33, I-00078 Monteporzio Catone, Italy \\ ${ }^{8}$ Department of Physics and Astronomy, University of Southampton, Highfield, Southampton SO17 1BJ, UK \\ ${ }^{9}$ Raman Research Institute, C. V. Raman Avenue, Sadashivanagar, Bangalore 560080, India \\ ${ }^{10}$ Centre for Extragalactic Astronomy, Department of Physics, Durham University, South Road, Durham DH1 3LE, UK \\ ${ }^{11}$ INAF - Istituto di Astrofisica Spaziale e Fisica Cosmica di Milano, via A. Corti 12, 20133 Milano, Italy \\ ${ }^{12}$ Jodrell Bank Centre for Astrophysics, Department of Physics and Astronomy, University of Manchester, Manchester M13 9PL, UK \\ ${ }^{13}$ Jet Propulsion Laboratory, California Institute of Technology, Pasadena, CA 91109, USA
}

Accepted 2020 November 18. Received 2020 November 17; in original form 2020 October 9

\begin{abstract}
We report on the discovery of a new, transient ultraluminous X-ray source (ULX) in the galaxy NGC 7090. This new ULX, which we refer to as NGC 7090 ULX3, was discovered via monitoring with Swift during 2019-2020, and to date has exhibited a peak luminosity of $L_{\mathrm{X}} \sim 6 \times 10^{39} \mathrm{erg} \mathrm{s}^{-1}$. Archival searches show that, prior to its recent transition into the ULX regime, ULX3 appeared to exhibit a fairly stable luminosity of $L_{X} \sim 10^{38} \mathrm{erg} \mathrm{s}^{-1}$. Such strong long-time-scale variability may be reminiscent of the small population of known ULX pulsars, although deep follow-up observations with XMM-Newton and NuSTAR do not reveal any robust X-ray pulsation signals. Pulsations similar to those seen from known ULX pulsars cannot be completely excluded, however, as the limit on the pulsed fraction of any signal that remains undetected in these data is $\lesssim 20$ per cent. The broad-band spectrum from these observations is well modelled with a simple thin disc model, consistent with sub-Eddington accretion, which may instead imply a moderately large black hole accretor $\left(M_{\mathrm{BH}} \sim 40 \mathrm{M}_{\odot}\right)$. Similarly, though, more complex models consistent with the super-Eddington spectra seen in other ULXs (and the known ULX pulsars) cannot be excluded given the limited signalto-noise ratio of the available broad-band data. The nature of the accretor powering this new ULX therefore remains uncertain.
\end{abstract}

Key words: stars: black holes - stars: neutron -X-rays: binaries - X-rays: individual (NGC 7090 ULX3).

\section{INTRODUCTION}

The population of ultraluminous X-ray sources (ULXs) - X-ray binaries (XRBs) which exhibit luminosities in excess of $10^{39} \mathrm{erg} \mathrm{s}^{-1}$ (see Kaaret, Feng \& Roberts 2017 for a recent review) - is now generally understood to be primarily made up of compact objects accreting close to or above their Eddington limits. This is driven by both spectroscopic and timing observations. For sources that can be studied in detail, the broad-band spectra provided by NuSTAR (Harrison et al. 2013) are inconsistent with standard modes of subEddington accretion (e.g. Bachetti et al. 2013; Rana et al. 2015; Mukherjee et al. 2015; Walton et al. 2015b, 2017), confirming the indications seen previously based on lower energy data (e.g. Stobbart, Roberts \& Wilms 2006; Gladstone, Roberts \& Done 2009; Walton et al. 2011a), and are instead similar to the broad expectation

^E-mail: dwalton@ast.cam.ac.uk for super-Eddington accretion (emission from a hot and complex accretion disc; e.g. Shakura \& Sunyaev 1973; Abramowicz et al. 1988). In addition, powerful outflows have now been observed in a number of ULXs through blueshifted atomic features (Pinto, Middleton \& Fabian 2016; Pinto et al. 2017, 2020; Walton et al. 2016b; Kosec et al. 2018), as predicted for super-Eddington accretion. Furthermore, a growing number of ULXs are now being identified as X-ray pulsars, which must therefore be highly superEddington neutron star accretors (Bachetti et al. 2014; Fürst et al. 2016; Israel et al. 2017a,b; Carpano et al. 2018; Sathyaprakash et al. 2019; Rodríguez Castillo et al. 2020; see also Brightman et al. 2018). ${ }^{1}$ As the best local examples of (relatively) sustained super-

${ }^{1}$ In addition to these more traditional ULX pulsars, there are also a small number of neutron star Be/XRBs that occasionally and briefly peak at $\sim 10^{39} \mathrm{erg} \mathrm{s}^{-1}$ during their largest outbursts, for example, A 0538-66 (Skinner et al. 1982), Swift J0243.6+6124 (Wilson-Hodge et al. 2018; Tao et al. 2019), and RX J0209.6-7427 (Vasilopoulos et al. 2020b; Chandra et al. 2020). 


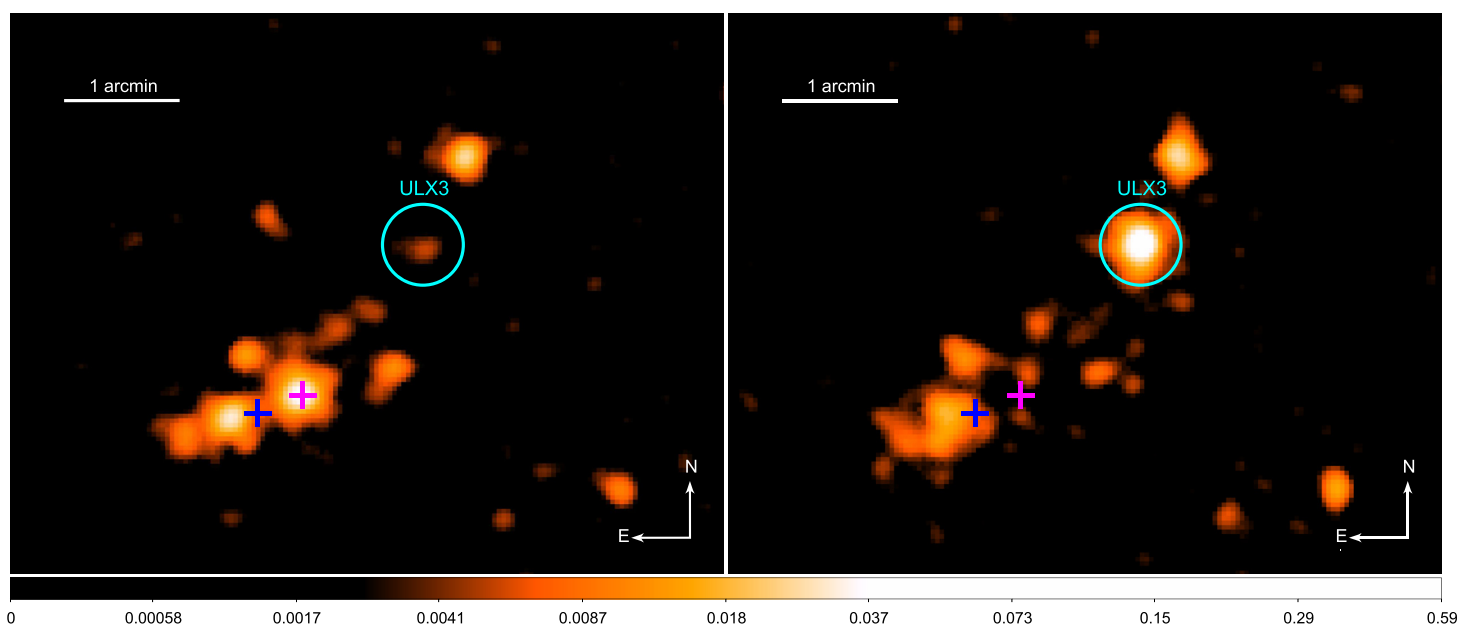

Figure 1. Swift-XRT images (0.3-10.0 keV) of NGC 7090, produced with the online XRT pipeline (Evans et al. 2009), taken over the lifetime of the mission prior to our monitoring campaign (left) and during our campaign (spread throughout 2019-2020; right). Both panels have been smoothed with a 4-pixel Gaussian for clarity and the colour bar across the bottom - common to both panels - is in units of $\mathrm{ct} \mathrm{ks}^{-1}$. The position of the newly discovered ULX3, which we report here, is shown with the cyan circle. The blue cross (further to the east) shows the position of ULX1 (e.g. Song et al. 2020), seen in XMM-Newton data but not obviously in either of the Swift images (note that this is distinct from the persistent emission seen by Swift even further to the east of ULX1), and the magenta cross (further to the west) shows the position of ULX2 (e.g. Liu et al. 2019), seen in the earlier Swift data but not during our more recent campaign.

Eddington accretion, these sources may have significant relevance for the growth of supermassive black holes $(\mathrm{BH})$ in the early universe, given the constraints placed by observations of $\sim 10^{9} \mathrm{M}_{\odot}$ BHs when the universe was less than a Gyr old (e.g. Mortlock et al. 2011; Bañados et al. 2018), so they are potentially a key population to understand.

ULXs are known to be associated with recent star formation (e.g. Swartz et al. 2011; Mineo, Gilfanov \& Sunyaev 2012; Lehmer et al. 2019), and are therefore generally thought of as high-mass X-ray binary (HMXB) analogues. Indeed, where it has been possible to place robust constraints, either via optical spectroscopy or X-ray timing, ULX stellar counterparts have generally been found to be massive (e.g. Motch et al. 2014; Bachetti et al. 2014; Heida et al. 2015, 2016, 2019). HMXBs are generally persistent sources, as the $\mathrm{BH}$ or neutron star feeds from the stellar wind of its massive companion (see Martínez-Núñez et al. 2017 for a recent review). However, it is challenging to produce the high accretion rates needed to explain the observed luminosities from ULXs purely via windfed accretion, and so accretion via Roche lobe overflow - the primary mechanism by which low-mass X-ray binaries (LMXBs) accrete (e.g. Verbunt 1993) - may be required despite their apparent connection with the HMXB population (e.g. Fürst et al. 2018; El Mellah, Sundqvist \& Keppens 2019). This may be related to specific periods in the evolution of the binary system, during which mass can be transferred from the stellar companion on its thermal timescale (e.g. King et al. 2001; Misra et al. 2020). Furthermore, as the archive of X-ray data continues to grow, and along with it the number of nearby galaxies with multiple observing epochs, an increasing number of new/transient ULXs are being reported (e.g. Soria et al. 2012; Middleton et al. 2012, 2013; Pintore et al. 2018, 2020; Earnshaw et al. 2019a, 2020; van Haaften et al. 2019; Brightman et al. 2020). The high-amplitude long-term variability

Although this is distinct behaviour from the known ULX pulsars, which spend extended periods at highly super-Eddington luminosities, these may be interesting objects in terms of connecting ULX pulsars to the sub-Eddington $\mathrm{X}$-ray pulsar population. seen from these systems is also generally difficult to explain in the context of persistent wind-fed accretion, and is also more typically seen in LMXBs. However, even if the mass transfer rate is relatively stable, such behaviour may still be possible for the magnetic neutron stars in ULX pulsars if the propeller effect is invoked (e.g. Tsygankov et al. 2016). Indeed, searching for sources that could be consistent with propeller transitions is a promising means to identify ULX pulsar candidates among the broader ULX population (e.g. Earnshaw, Roberts \& Sathyaprakash 2018; Song et al. 2020), so these highly variable ULXs are potentially of particular interest.

Here, we report on the discovery and characterization of a new, transient ULX in NGC $7090(z=0.00282)$, utilizing observations with the Neil Gehrels Swift Observatory (hereafter Swift; Gehrels et al. 2004), XMM-Newton (Jansen et al. 2001), NuSTAR (Harrison et al. 2013), and Chandra (Weisskopf et al. 2002). Throughout this work, we assume a distance to NGC 7090 of $D=9.5 \mathrm{Mpc}$ (based on the tip of the red giant branch method, Karachentsev, Kaisina \& Makarov 2018).

\section{NGC 7090 ULX3}

NGC 7090 was previously known to host two highly variable/transient ULXs (Walton et al. 2011b; Earnshaw et al. 2019b; Liu et al. 2019; Song et al. 2020). During a brief monitoring programme targeting this galaxy with Swift throughout 2019-2020, primarily intended to track the activity of the brighter of these two sources, we serendipitously discovered that a third, previously unknown ULX had appeared in the XRT data at RA $=21^{h} 36^{m} 22.74^{s}$, Dec. $=$ $-54^{\circ} 32^{\prime} 33.8^{\prime \prime}$ (see Section 4), which we refer to as NGC 7090 ULX3 (hereafter simply ULX3; see Fig. 1). We therefore triggered a deep $X M M-N e w t o n+N u S T A R$ target-of-opportunity observation (epoch $\mathrm{XN1}$ ) in order to investigate the spectral and temporal properties of this new source. This observation was performed on 2020 April 28; further details are given in Table 1. Both the XMM-Newton and NUSTAR data were reduced following standard procedures, as outlined below. 
Table 1. Details of the XMM-Newton, NuSTAR, and Chandra X-ray observations of NGC 7090 considered in this work.

\begin{tabular}{lcccc}
\hline Epoch & Mission & OBSID & $\begin{array}{c}\text { Start } \\
\text { date }\end{array}$ & $\begin{array}{c}\text { Good } \\
\text { exposure }\end{array}$ \\
\hline XN1 & NuSTAR & $\begin{array}{c}\text { 2020 Observations } \\
80501321002\end{array}$ & $2020-04-28$ & 122 \\
& XMM-Newton & 0852050201 & $2020-04-29$ & $82 / 100$ \\
& Archival data & & \\
X1 & XMM-Newton & 0200230101 & $2004-04-08$ & - \\
X2 & XMM-Newton & 0200230201 & $2004-05-13$ & $6 / 11$ \\
C1 & Chandra & 7060 & $2005-12-18$ & 26 \\
C2 & Chandra & 7252 & $2006-04-10$ & 31 \\
X3 & XMM-Newton & 0503460101 & $2007-10-05$ & $6 / 8$ \\
\hline Note: & & &
\end{tabular}

Note: ${ }^{a}$ Exposures are given in ks, and for XMM-Newton are listed for the EPIC-pn/MOS detectors after background flaring has been excised.

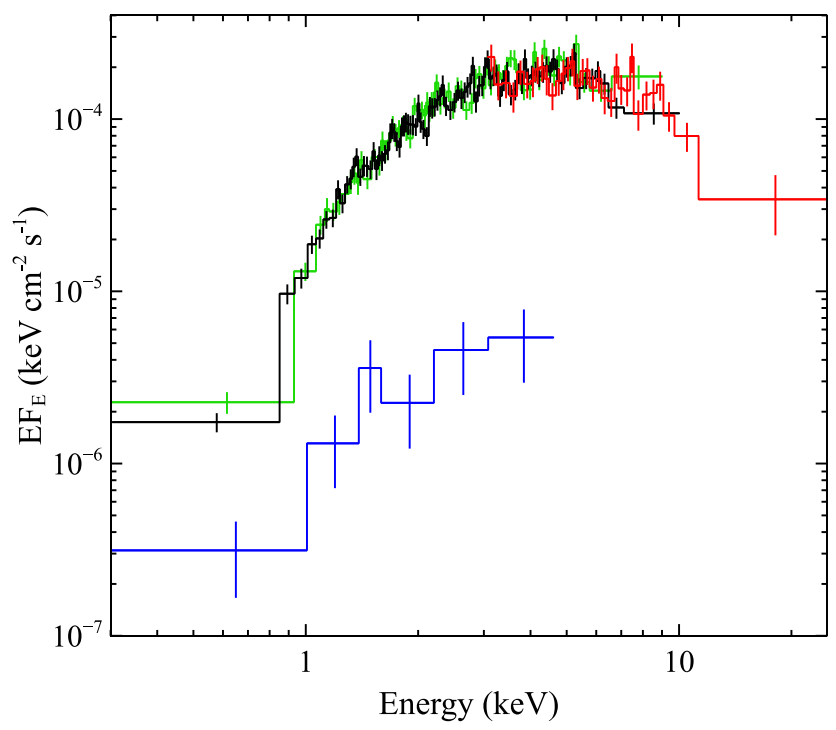

Figure 2. The broad-band XMM-Newton+NuSTAR X-ray spectrum of NGC 7090 ULX3 taken in 2020 (epoch XN1). The data have been unfolded through a model that is constant with energy, and rebinned for visual clarity. For epoch XN1, the XMM-Newton EPIC-pn and EPIC-MOS data are shown in black and green, respectively, and the NUSTAR FPM data are shown in red. We also show a comparison with one of the low-flux archival observations, epoch C1, with the ACIS-S data in blue.

\subsection{Observations and data reduction}

The NUSTAR data were reduced with the NUSTAR Data Analysis Software (NUSTARDAS) v1.9.2, and NUSTAR calibration data base v20190627. First the unfiltered event files for both focal plane modules (FPMA and FPMB) were cleaned with NUPIPELINE, using the standard depth correction to reduce the internal high-energy background and excluding passages through the South Atlantic Anomaly. The data for both modules were corrected to the solar barycentre using the DE200 solar ephemeris. Source products and their associated instrumental response files were then extracted for each module using circular regions of radius $30 \mathrm{arcsec}$ with NUPRODUCTS. For all the data sets considered here, their associated backgrounds were estimated from larger regions of blank sky on the same chip as ULX3. In order to maximize the signal-to-noise ratio $(\mathrm{S} / \mathrm{N})$, we use both the standard 'science' data (mode 1) and the 'spacecraft science' data (mode 6; see Walton et al. 2016c); the mode 6 data provide $\sim 14$ per cent of the total NUSTAR exposure quoted in Table 1 . Finally, given the moderate $\mathrm{S} / \mathrm{N}$ of the data for the individual focal plane modules, we combined their spectra using ADDASCASPEC; we also note that this observation was not affected by the recently identified tear in the thermal blanket (Madsen et al. 2020). The NUSTAR data provide constraints up to $\sim 20-25 \mathrm{keV}$ in this case; above these energies there is no significant detection of ULX3 above the background level.

The $X M M-N e w t o n$ data were reduced using the $X M M-N e w t o n$ Science Analysis System (SAS v18.0.0). Raw observation files were cleaned using EPCHAIN and EMCHAIN for the EPIC-pn and EPICMOS detectors, respectively (Strüder et al. 2001; Turner et al. 2001). The cleaned event files for EPIC-pn, which has the best time resolution of the EPIC detectors $(73.4 \mathrm{~ms}$ in the full frame mode used here), were corrected to the solar barycentre using the DE200 solar ephemeris, similar to the NUSTAR data. Source products were extracted from the cleaned event files with XMMSELECT from a circular region of radius 30 arcsec; periods of high background were removed as standard, but only occur at the very end of the observation. As recommended, we only considered single and double patterned events for EPIC-pn (PATTERN $\leq 4)$ and single to quadruple patterned events for EPIC-MOS (PATTERN $\leq 12)$. The instrumental response files were generated using ARFGEN and RMFGEN for each of the EPIC detectors. After performing the reduction separately for the two EPIC-MOS units, we also combined these data using ADDASCASPEC.

\subsection{Spectroscopy}

The broad-band X-ray spectrum extracted from the $2020 X M M-$ Newton + NuSTAR data set is shown in Fig. 2. The data have a distinctly thermal appearance, peaking at $\sim 5 \mathrm{keV}$ before falling away with a steep spectrum at higher energies, qualitatively similar to the other ULXs observed by NUSTAR to date (e.g. Walton et al. 2018 b,c). The flux from ULX3 is stable throughout the observation (see Section 2.3), so we apply some simple continuum models to the time-averaged broad-band data using XSPEC (v12.10.1s; Arnaud 1996). All the individual data sets from epoch XN1 are rebinned to a minimum $\mathrm{S} / \mathrm{N}$ of 5 per energy bin, and the data are fit by reducing the $\chi^{2}$ statistic. As is standard, we allow for normalization constants to vary between the data sets to account for residual cross-calibration issues, fixing EPIC-pn to unity; for the models that successfully fit the data, these are all within normal ranges (Madsen et al. 2015). Neutral absorption is modelled with TBABS, combining the crosssections of Verner et al. (1996) and the solar abundance set of Wilms, Allen \& McCray (2000). In all of the models considered here, we include absorption component that corresponds to the Galactic column $\left(2 \times 10^{20} \mathrm{~cm}^{-2}\right.$; HI4PI Collaboration et al. 2016), and also separately allow for absorption local to the source that is free to vary.

We first apply an absorbed power-law continuum. However, this provides a very poor fit to the data, with $\chi^{2}=635$ for 326 degrees of freedom (d.o.f.). Given the thermal appearance of the data, we therefore also try a simple accretion disc model, using DISKBB (Mitsuda et al. 1984). This assumes a standard thin accretion disc, based on the model of Shakura \& Sunyaev (1973). In contrast to the power-law continuum, this simple model actually provides a very good fit to the data, with $\chi^{2} /$ d.o.f. $=341 / 326$. Given the evidence that most ULXs are likely super-Eddington accretors, we also fit the DISKPBB accretion disc model (Mineshige et al. 1994). This allows the radial temperature index, $p$, to vary as an additional free parameter (defined such that $T(r) \propto r^{-p}$ ), and is often used to approximate superEddington accretion discs. These are expected to have a significant scale height and, as discussed by Abramowicz et al. (1988), should 
Table 2. Best-fitting parameters obtained for the simple continuum models applied to the broad-band spectrum observed from ULX3 (epoch XN1).

\begin{tabular}{lccc}
\hline Model: & Power law & DISKBB & DISKPBB \\
\hline$N_{\mathrm{H}}\left(10^{21} \mathrm{~cm}^{-2}\right)$ & $16.5 \pm 0.1$ & $7.9 \pm 0.5$ & $7.4 \pm 1.2$ \\
$\Gamma$ & $2.05 \pm 0.06$ & - & - \\
$T_{\text {in }}(\mathrm{keV})$ & - & $1.87 \pm 0.07$ & $1.8_{-0.1}^{+0.2}$ \\
$p$ & - & - & $0.8 \pm 0.1$ \\
Norm $\left(10^{-4}\right)$ & $1.8_{-0.2}^{+0.1}$ & $23 \pm 3$ & $28_{-11}^{+21}$ \\
$\chi^{2} /$ d.o.f. & $635 / 326$ & $341 / 326$ & $341 / 325$ \\
\hline
\end{tabular}

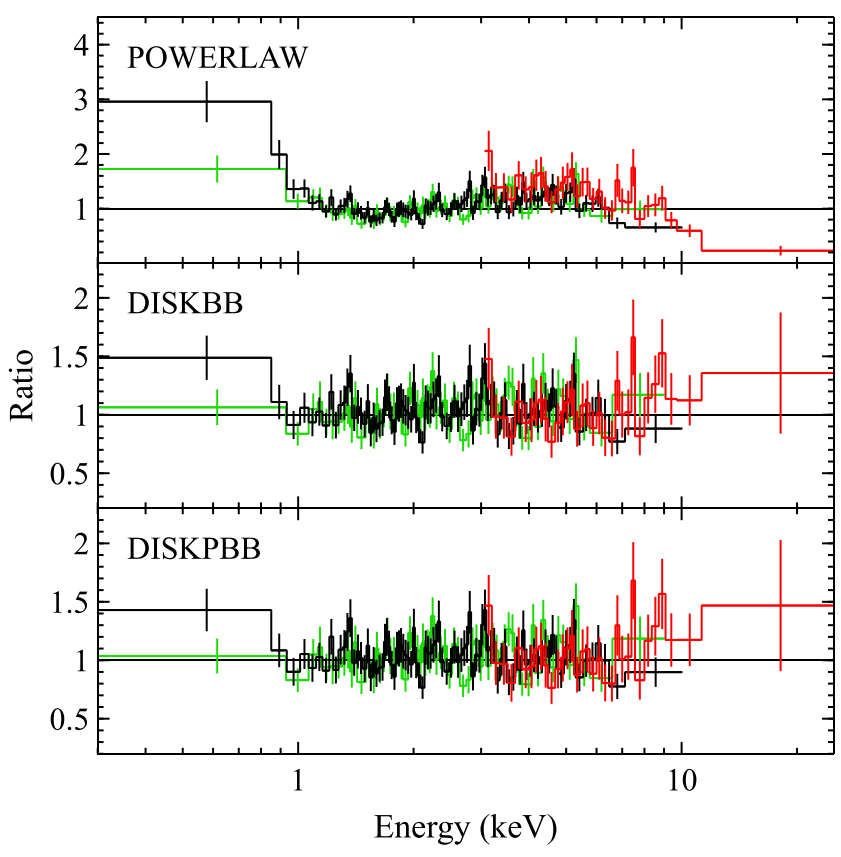

Figure 3. Data/model ratios for the simple continuum models applied to the broad-band data set (epoch XN1) for ULX3 (Section 2.2). The colours have the same meanings as in Fig. 2.

be characterized by $p \sim 0.5$ (as opposed to the thin disc solution, for which $p=0.75$ ). Although the DISKPBB model also fits the data very well, with $\chi^{2} /$ d.o.f. $=341 / 325$, this offers a negligible improvement over the simpler DISKBB model despite the extra free parameter $\left(\Delta \chi^{2} \sim 0.5\right)$. Indeed, when allowed to vary, we find that $p$ is consistent with the standard thin disc solution in this case. The results obtained with these models are given in Table 2, and the data/model ratios for each of these fits are shown in Fig. 3.

Following previous work on ULX spectroscopy, we try a variety of more complex models, but find that these are not required by the data from epoch XN1. Below $10 \mathrm{keV}$, high S/N ULX spectra typically require the presence of two thermal disc-like components, typically with temperatures $\sim 0.3$ and $\sim 3 \mathrm{keV}$ (e.g. Stobbart et al. 2006; Gladstone et al. 2009). The hotter component (also seen here) is likely associated with the innermost accretion flow, while the lower temperature component is potentially associated with either the outer accretion flow or the photosphere of a strong, super-Eddington wind (e.g. King \& Pounds 2003). However, adding a second, lowtemperature thermal component to the baseline DISKBB model only improves the fit by $\Delta \chi^{2}=3$ for two more free parameters. It is worth noting, though, that the line-of-sight absorption column towards ULX3 is quite high in this case, and so the presence of

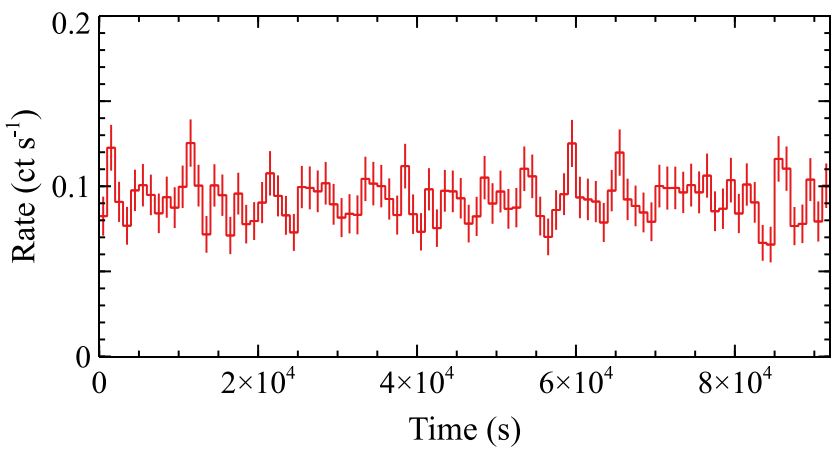

Figure 4. The 0.3-10.0 keV EPIC-pn light curve of NGC 7090 ULX3 during epoch XN1, shown with 1 ks time bins.

an additional low-temperature emission component could easily be masked by absorption.

At higher energies, all of the ULXs observed by NUSTAR with good $\mathrm{S} / \mathrm{N}$ to date show evidence for additional continuum emission above $\sim 10 \mathrm{keV}$ when the lower energy data are fit with accretion disc models (e.g. Walton et al. 2014, 2015b, 2018c; Mukherjee et al. 2015; Fürst et al. 2017). In the case of the ULX pulsars, this emission is known to be associated with the central, magnetically channelled accretion columns (Brightman et al. 2016; Walton et al. 2018a,b), while for non-magnetic neutron star/BH ULXs this emission would presumably arise from a Compton-scattering corona. Adding an additional high-energy component to represent either of these possibilities - using the SIMPL convolution model (Steiner et al. 2009) for the power-law emission from a corona and the template for ULX pulsar accretion columns adopted in Walton et al. (2020, a CUTOFFPL component with $\Gamma=0.59$ and $E_{\text {cut }}=7.9 \mathrm{keV}$ ) - also results in a negligible improvement in the fit, with $\Delta \chi^{2}=1$ in both cases. However, the high-energy $(E>10 \mathrm{keV}) \mathrm{S} / \mathrm{N}$ is significantly lower in this case than for any of the sources in the NUSTAR sample discussed in Walton et al. (2018c), where evidence for the extra high-energy component was ubiquitously seen. In the case of the SIMPL model, we find an upper limit on the scattered fraction (which acts as an effective normalization for the power-law flux) of $f_{\text {sc }}<32$ per cent, which is not dissimilar to the values seen in other ULXs when fit with a similar model (typical values are a few 10 s of per cent, e.g. Walton et al. 2015b).

For the DISKBB model, which provides the simplest explanation of the broad-band data, we compute the observed flux in the 0.3$10 \mathrm{keV}$ band, for comparison with archival data sets (Section 3), and absorption corrected luminosities in the $0.3-10.0$ and $0.3-30.0 \mathrm{keV}$ bands. We find $F_{\text {obs, } 0.3-10}=(4.4 \pm 0.1) \times 10^{-13} \mathrm{erg} \mathrm{cm}^{-2} \mathrm{~s}^{-1}$, $L_{\text {int }, 0.3-10}=(6.0 \pm 0.2) \times 10^{39} \mathrm{erg} \mathrm{s}^{-1}$, and $L_{\text {int, } 0.3-30}=(6.4 \pm 0.2) \times$ $10^{39} \mathrm{erg} \mathrm{s}^{-1}$ (assuming isotropic emission). Similar to other ULXs, the vast majority of the broad-band flux is emitted below $10 \mathrm{keV}$.

\subsection{Timing analysis}

The X-ray light curve from the EPIC-pn detector for epoch XN1 is shown in Fig. 4; overall, the source flux appears to be relatively stable during these observations. We therefore limit our variability analysis to a search for pulsations in order to explore the possibility that ULX3 is a new member of the ULX pulsar population.

We use several strategies to look for pulsations - ranging from general pulsation searches to deeper searches based on the properties of known ULX pulsars - utilizing the pulsar timing tools included in HENDRICS (Bachetti 2018). We focus primarily on the data from 
the EPIC-pn camera, as this has both the highest count rates and the best temporal resolution of the EPIC detectors, and investigate candidate pulsations below a Nyquist frequency corresponding to the 73-ms frame time of EPIC-pn. Our goal is to find pulsations whose frequency might be changing quickly during our observation due to the intrinsic spin-up/down (Israel et al. 2017a) or to orbital motion (e.g. Bachetti et al. 2014; Rodríguez Castillo et al. 2020). The first effect should give rise to an approximately constant acceleration (i.e. a linear change) of the pulse frequency during the observation.

For this analysis, we maximize the $\mathrm{S} / \mathrm{N}$ by running a Fourier-space accelerated search (Ransom, Eikenberry \& Middleditch 2002) on the whole light curve using the HENACCELSEARCH tool. The range in frequency derivatives we search formally depends on the frequency being searched, but at a central frequency of $1 \mathrm{~Hz}$ we search a range of $\pm 5 \times 10^{-8} \mathrm{~Hz} \mathrm{~s}^{-1}$ with a resolution of $2.5 \times 10^{-10} \mathrm{~Hz} \mathrm{~s}^{-1}$. This task uses standard 'detection levels' based on the $\chi^{2}$ distribution (Leahy et al. 1983). To account for the different sensitivity response across a given frequency bin (which leads to a drop of sensitivity as the pulsation frequency departs from the central frequency and approaches the edge of the bin), the tool initially considers all frequencies with variability powers in excess of 36 per cent of the power level corresponding to a 1 per cent false-alarm probability as 'candidate' pulsations. These candidates are then investigated with more sensitive $Z_{n}^{2}$ search (Buccheri et al. 1983). Additionally, we also run the accelerated search on shorter, overlapping intervals of the light curve (down to $50 \mathrm{ks}$ ) to look for non-linear frequency changes (from orbital motion) and transient pulsations. Motivated by the generally hard pulsed spectra in ULX pulsars (e.g. Brightman et al. 2016; Walton et al. 2018b), we repeat these searches both on the full event lists and in the $2.5-12 \mathrm{keV}$ energy range. Since most ULX pulsars have quasi-sinusoidal profiles, we do not perform any harmonic summing in the power density spectra calculated by HENACCELSEARCH and we limit the number $n$ of harmonics in the $Z_{n}^{2}$ searches to 2 .

There are a few candidate frequencies (at 0.88 and $4.36 \mathrm{~Hz}$ for the $2.5-12 \mathrm{keV}$ bandpass and $6.4 \mathrm{~Hz}$ for the full data set) that slightly exceeded the nominal detection level. Unfortunately, these time-scales are all too fast for independent verification with the EPIC-MOS detectors, and the NUSTAR data are also unable to independently confirm whether any of them represent a real signal (although the NUSTAR limits are less stringent owing to its lower count rates). Therefore, given the lack of consistency between the full and the harder bands, and the lack of independent verification with any other available detector, we do not consider any of these potential signals to be robust, bona-fide detections. Nevertheless, we report them in case one of these frequencies is seen again in future observations with similar significance (with a blind search), as this would potentially transform one of these marginal cases into a more believable pulsation signal.

Given the lack of a robust pulsation detection, we calculate the upper limit on the pulsed fraction any pulsed signal could have using HENZN2VSPF, focusing on the $0.01-7 \mathrm{~Hz}$ frequency range. HENZN2VSPF simulates data sets using the same good-time intervals (GTIs) and total number of events in each GTI as seen in the real data, and uses rejection sampling to modulate the events with stronger and stronger pulsations. For each simulated data set, the tool calculates the $Z_{2}^{2}$ statistics and produces a $Z_{2}^{2}$ versus pulsed fraction plot. We simulate 100 data sets with increasing pulsed fraction, and determine the point where the $Z_{2}^{2}$ reaches $\sim 40$. This roughly corresponds to a $3 \sigma$ statistical detection, and thus gives the equivalent upper limit on the pulsed fraction permitted by the real data. We find limits on the pulsed fraction of $\sim 20$ per cent for the full data set and $\sim 40$ per cent for the energy- or time-selected intervals. We note that these values are similar to the pulsed fractions inferred for the marginal pulsation candidates listed above, confirming again that they should be treated with caution.

\section{ARCHIVAL DATA AND LONG-TERM VARIABILITY}

In addition to the new XMM-Newton + NuSTAR observation presented here, there are also three archival observations of NGC 7090 with XMM-Newton, and two with Chandra, which we also consider to provide contextual information over a longer time-scale; these are spread over the period 2004-2007 (see Table 1). The two Chandra observations, both taken with the ACIS-S detector (Garmire et al. 2003), were reduced with CIAO v4.11 and its associated calibration files. Cleaned event files were generated with the CHANDRA_REPRO script. ULX3 is clearly detected in both Chandra observations, ${ }^{2}$ albeit at much lower fluxes, and so source spectra and instrumental response files were extracted with the SPECEXTRACT script from circular regions of radius 2 arcsec. For the first $X M M-N e w t o n$ observation (epoch X1), the entire exposure suffered from severe background flaring, so we do not make any use of these data. ULX3 is clearly detected in the second observation (epoch X2), ${ }^{2}$ and appears to be marginally detected in the third (epoch X3). As with the Chandra data, the source fluxes are significantly lower than epoch XN1, so we extract source spectra largely as outlined above, but using a smaller source region (radius 15 arcsec). The only difference is that for epoch $\mathrm{X} 3$, the position of ULX3 fell on a bad row for the EPIC-MOS1 detector, so in this case we only utilize the data from the EPIC-pn and EPIC-MOS2 detectors.

Of these archival data sets, epoch $\mathrm{C} 1$ has the highest $\mathrm{S} / \mathrm{N}$, and so we show this in comparison to the high-flux data in Fig. 2. Over the more limited bandpass covered, the observed spectrum is still quite hard, although this is not surprising given the reasonably substantial absorption column inferred from the epoch XN1 data. To model these low-flux data, given the low $\mathrm{S} / \mathrm{N}$, we group them to 1 count per bin and fit them by reducing the Cash (1979) statistic. We also fix the level of absorption to that found previously (i.e. $N_{\mathrm{H}}=7.9 \times 10^{21} \mathrm{~cm}^{-2}$ ). For both power-law and accretion disc continuum models (assuming a thin disc for simplicity), we find that the data from epochs $\mathrm{C} 1$, $\mathrm{C} 2, \mathrm{X} 2$, and $\mathrm{X} 3$ can all be fit with a common spectral shape; with the former we find $\Gamma=2.2 \pm 0.4$ and with the latter we find $T_{\text {in }}=$ $0.9 \pm 0.2 \mathrm{keV}$. We use the latter to compute observed fluxes in the 0.3-10 keV band for each of these epochs (the fluxes computed with the power-law continuum are systematically $\sim 20$ per cent higher, but are ultimately in good statistical agreement with the DISKBB fluxes). These are typically $\sim 10^{-14} \mathrm{erg} \mathrm{cm}^{-2} \mathrm{~s}^{-1}$ for each of these epochs, corresponding to luminosities of $\sim 10^{38} \mathrm{erg} \mathrm{s}^{-1}$ (again, assuming isotropic emission). Interestingly, with the DISKBB model, we find the normalizations are all consistent at the 90 per cent level, and the bestfitting values are all in the range $1-2 \times 10^{-3}$, broadly similar to the value seen from epoch XN1. Indeed, if we fit the low-flux data with a common normalization, instead of a common disc temperature, we find that the normalization is $1.4_{-0.9}^{+2.1} \times 10^{-3}$, while the bestfitting disc temperatures vary between $T_{\text {in }} \sim 0.7-0.9 \mathrm{keV}$. The DISKBB normalization is proportional to $\left(R_{\text {in }} / D\right)^{2} \cos (i)$, where $R_{\text {in }}$ is the inner radius of the disc, $D$ is the distance to the source, and $i$ is the disc

\footnotetext{
${ }^{2}$ The low-flux detections of ULX3 are included in the CSC2 and 4XMM catalogues (Evans et al. 2020; Webb et al. 2020) under the identifiers 2CXO J213622.6-543234 and 4XMM J213622.4-543233.
} 


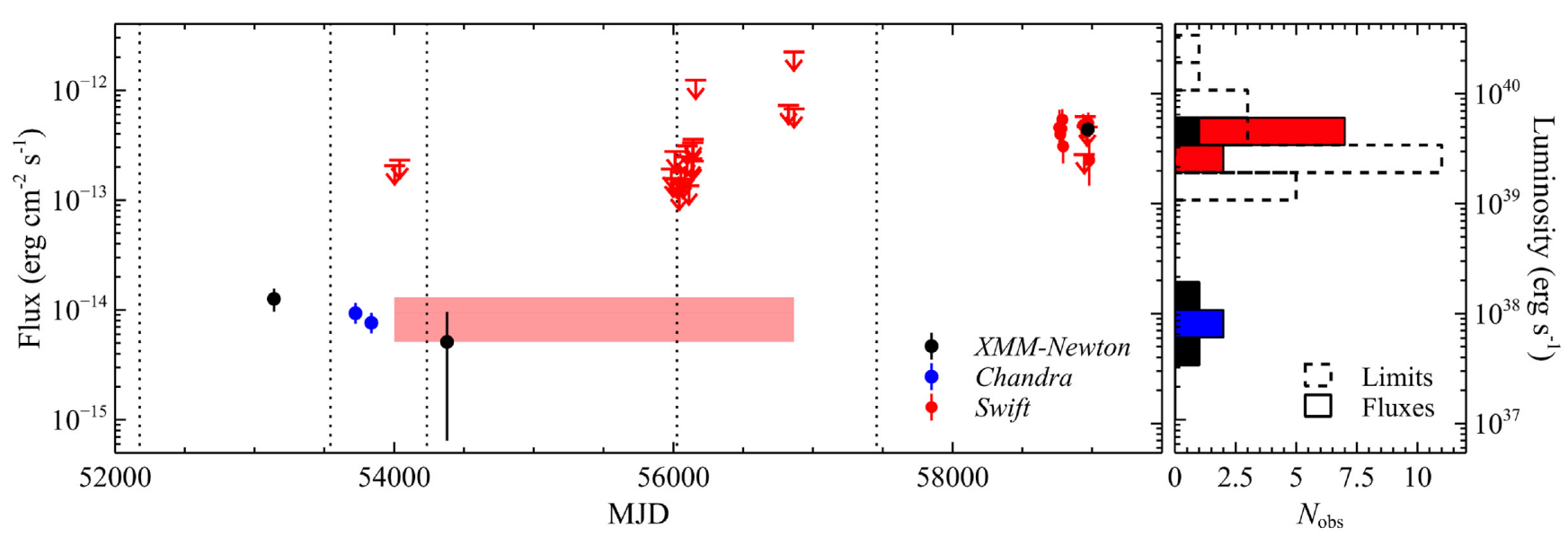

Figure 5. The long-term 0.3-10 keV X-ray light curve of ULX3 (left-hand panel), and the corresponding flux distribution (right-hand panel). Data from $X M M-N e w t o n, C h a n d r a$, and Swift are shown in black, blue, and red, respectively. The pink shaded region corresponds to the average flux seen stacking all of the archival Swift observations. ULX3 shows both high- and low-flux states, separated by more than an order of magnitude, similar to known ULX pulsars. The timing of the HST observations considered in Section 4 are indicated with vertical dotted lines.

Table 3. Details of the HST observations covering the position of ULX3. Magnitudes of the candidate optical counterparts are apparent instrumental Vega magnitudes computed with DOLPHOT.

\begin{tabular}{lcccccc}
\hline Inst & Prop ID & Obs date & Filter & Exp time (s) & Src 1 & Src 2 \\
\hline WFPC2 & 09042 & $2001-09-24$ & F450W & 460 & $24.73 \pm 0.18$ & $>25.3$ \\
WFPC2 & 09042 & $2001-09-24$ & F814W & 460 & $22.60 \pm 0.06$ & $>24.6$ \\
ACS & 10416 & $2005-06-23$ & F625W & 2508 & $23.227 \pm 0.010$ & $26.8 \pm 0.1$ \\
ACS & 10416 & $2005-06-23$ & F658N & 7496 & $22.94 \pm 0.03$ & $25.9 \pm 0.4$ \\
WFPC2 & 10889 & $2007-05-14 / 17$ & F814W & 6000 & $22.528 \pm 0.012$ & $24.41 \pm 0.05$ \\
ACS & 12546 & $2012-04-09$ & F606W & 900 & $23.466 \pm 0.014$ & $27.6 \pm 0.4$ \\
ACS & 12546 & $2012-04-09$ & F814W & 900 & $22.480 \pm 0.013$ & $24.97 \pm 0.08$ \\
WFC3 & 14095 & $2016-03-08$ & F110W & 298 & $21.833 \pm 0.018$ & $22.05 \pm 0.02$ \\
WFC3 & 14095 & $2016-03-08$ & F128N & 903 & $21.88 \pm 0.03$ & $22.11 \pm 0.03$ \\
\hline
\end{tabular}

inclination (see below), and so the data are formally consistent with being dominated by a standard thin disc which has a constant inner radius across all of the observing epochs to date (although we stress that the uncertainties are large for the low-flux data).

We also extract the Swift $0.3-10.0 \mathrm{keV}$ light curve from the online XRT pipeline (Evans et al. 2009) in order to build a more complete picture of the flux history of ULX3. In addition to the 12 observations taken in 2019/20 as part of our recent program (typically $\sim 3-4$ ks exposures), Swift has also observed NGC 7090 on a further 21 occasions (typically 2-4 ks exposures) between 2006-2014 (spanning MJD 54000-57000). Although there appears to be a weak detection in the integrated XRT image from all of the observations that were taken prior to our more recent campaign (see Fig. 1), ULX3 is not significantly detected in any of these individual Swift observations. The XRT count rates (and their limits) are converted to fluxes for comparison with the other data sets assuming the spectral forms discussed above; for our recent monitoring campaign we use the spectral form found for epoch $\mathrm{XN1}$, while for the archival data we use the spectral form found for epochs $\mathrm{C} 1, \mathrm{C} 2, \mathrm{X} 2$, and $\mathrm{X} 3$. Then, combining the $0.3-10.0 \mathrm{keV}$ flux from epoch $\mathrm{XN} 1$ with all of these fluxes, we construct the long-term lightcurve of ULX3 shown in Fig. 5.

Although the coverage is quite sparse, the detections provided by XMM-Newton, Chandra, and Swift show evidence for highamplitude (more than an order of magnitude) long-term variability that is consistent with a bi-modal flux distribution. To further test this potential bi-modality, we also extract the spectrum for the weak detection seen in the integrated archival Swift data in Fig. 1 (i.e. prior to MJD 58000). Modelling this with the same spectral form as seen in the rest of the low-flux archival data sets (a thin disc with $T_{\text {in }}=$ $0.9 \mathrm{keV}$ ), again binning the data to 1 count per bin and reducing the Cash statistic, we find an average flux of $\sim 10^{-14} \mathrm{erg} \mathrm{cm}^{-2} \mathrm{~s}^{-1}$ for these data, consistent with the rest of the low-flux epochs. Although we use all of the Swift data taken prior to 2020, this is dominated by a dense period of monitoring of NGC 7090 around MJD 56000, $\sim 4-5$ yr after the last archival XMM-Newton observation. This flux level does therefore appear to represent a fairly stable baseline for ULX3 prior to the observations obtained in 2020.

\section{OPTICAL COUNTERPARTS}

As NGC 7090 has been observed on several occasions by the Hubble Space Telescope (HST), we also perform a search for any optical counterparts to ULX3 (see Table 3 for a list of the observations used here). For this analysis, we focus on the imaging data from Chandra, and extract a combined image from the two Chandra observations using the CIAO task REPROJECT_OBS.

In order to register the various images to a common coordinate system, we produce source lists for both Chandra and HST using WAVDETECT and SEXTRACTOR for Chandra and HST, respectively (adopting fairly standard source detection thresholds in each case), 
each of which are matched against the Gaia DR2 source catalogue 3 (Gaia Collaboration et al. 2018). For Chandra, the image transformation is determined using WCSMATCH and then applied to the combined image using WCSUPDATE, both part of CIAO. The transformation is determined by initially matching sources within a 2 arcsec radius, and then iteratively updating the astrometric solution to keep only those that match within a radius of 0.5 arcsec once the transformation is applied; this results in five matches with the Gaia catalogue (note that none of these are the ULX in question), and leaves a residual uncertainty on the astrometric solution of $0.26 \operatorname{arcsec}(1 \sigma$ confidence). The position of ULX3 in the updated Chandra image is $\mathrm{RA}=21^{h} 36^{m} 22.74^{s}$, Dec. $=-54^{\circ} 32^{\prime} 33.8^{\prime \prime}$. This has a statistical precision of $0.09 \mathrm{arcsec}$, giving a total $3 \sigma$ uncertainty on the position of ULX3 of $0.83 \mathrm{arcsec}$ (combining the statistical and astrometric uncertainties in quadrature). Although we do not repeat this analysis for the XMM-Newton data, we note that there are no other X-ray sources detected by Chandra within 50 arcsec, and that the raw $X M M-N e w t o n$ centroid position from epoch $\mathrm{XN1}$ is in excellent agreement with the Chandra position given above. For HST, we use the 'Match to star positions' tool in the STARLINK/GAIA software to update the astrometric solutions of the drizzled images retrieved from the Hubble Legacy Archive. We find 7-12 good matches in each image. The resulting astrometric uncertainties are smaller than $0.05 \operatorname{arcsec}(1 \sigma$ confidence) for all images and thus provide a negligible contribution to the localization uncertainty of the ULX.

We detect two potential counterparts in the HST images. One is located on the edge of the error circle and clearly detected in all images. The second is located closer to the centre of the error circle but only clearly visible in the most recent, WFC3 near-IR images. Both sources are indicated in the bottom right panel in Fig. 6.

We obtain point spread function photometry of the two candidate counterparts with DOLPHOT (Dolphin 2000). Following the recommendations in the manual, we use the $\mathrm{c} 0 \mathrm{~m}$ and $\mathrm{c} 1 \mathrm{~m}$ (WFPC2) and flt (ACS and WFC3) images for the photometric analysis. We use the recommended parameter settings for each instrument, with fitsky $=2$. The computed instrumental Vega magnitudes for the sources indicated in Fig. 6 are listed in Table 3. Source 1 is clearly detected in all epochs and filters; source 2 is (sometimes marginally) detected in all but the first epochs. We determine the limiting magnitude for the 2001 WFPC2 observations using the fakestars routine in DOLPHOT, by simulating and retrieving 2000 stars with instrumental magnitudes in the range of 22-27 around the position of the ULX. We adopt the magnitude where 90 per cent of simulated stars are still detected with $\mathrm{S} / \mathrm{N} \geq 5$ as our limiting magnitude. Source 1 does not show variability in the F814W band, the only filter for which multiple epochs are available, while source 2 appears to be somewhat variable; unfortunately all F814W observations were obtained prior to the last Swift observation (in 2014) where the ULX was undetected, so we cannot investigate whether a change in the optical properties of the source accompanied the increase in the X-ray luminosity. We also subtract an appropriately scaled version of the ACS/F625W image from the ACS/F658N image to search for excess $\mathrm{H} \alpha$ emission associated with the ULX. However, we do not detect point-like excess $\mathrm{H} \alpha$ emission associated with either of the candidate counterparts in the continuum-subtracted image. The ULX does lie in a region with faint extended $\mathrm{H} \alpha$ emission, but this appears to be part of a larger structure not necessarily associated with the ULX itself.

${ }^{3}$ Unfortunately there are not enough Chandra sources in the relatively small field of view of the $H S T$ observations to directly register the images, hence our use of Gaia DR2 as an intermediary.

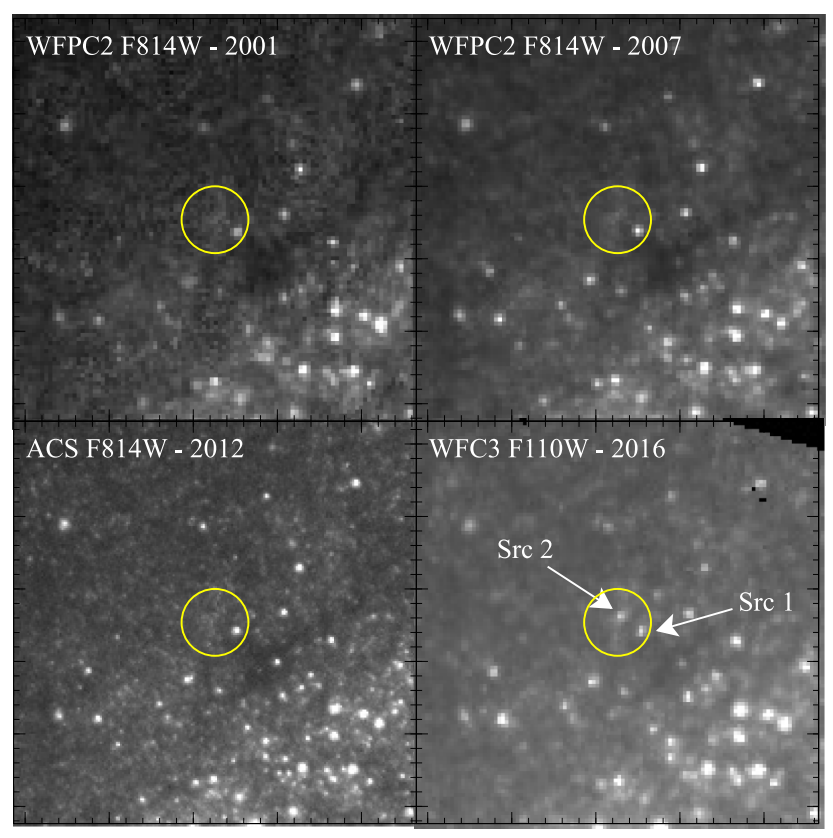

Figure 6. Drizzled HST images in the F814W and F110W filters. Each panel shows a $10 \operatorname{arcsec} \times 10 \operatorname{arcsec}$ region around the location of the ULX. North is up, and East to the left. The yellow circles indicate the 0.83 arcsec radius, $3 \sigma$ confidence localization of the ULX.

The Galactic foreground extinction in the direction of NGC 7090 is low $\left(A_{V}=0.063\right.$, Schlafly \& Finkbeiner 2011), but given that it is an edge-on spiral galaxy and that the ULX appears to be located on one of the dust lanes, there must be local extinction as well. Assuming $N_{\mathrm{H}}=2.21 \times 10^{21} A_{V} \mathrm{~cm}^{-2}$ (Güver \& Özel 2009) and $N_{\mathrm{H}}$ $\approx 7.9 \times 10^{21} \mathrm{~cm}^{-2}$, we find $A_{V} \approx 3.6$. As part of this hydrogen column may be intrinsic to the $\mathrm{X}$-ray source we consider this value as an upper limit to the extinction of the optical emission. With $A_{V}$ in the range of $1-3.6$ and $m-M=29.9$, source 1 has absolute magnitudes and colours roughly consistent with a blue or yellow supergiant (potentially similar to NGC 7793 P13; Motch et al. 2014). Similar to other ULXs with blue counterparts, the emission could also be dominated by an irradiated accretion disc (e.g. Roberts et al. 2011; Tao et al. 2012), although we note again that all of the optical observations appear to have been taken while ULX3 was in its lower luminosity state $\left(L_{\mathrm{X}} \sim 10^{38} \mathrm{erg} \mathrm{s}^{-1}\right.$, as far as it is possible to tell from the limited temporal coverage; see Fig. 5). Compared to the range of absolute magnitudes observed in Galactic LMXBs in outburst (which often reach comparable X-ray luminosities to NGC 7090 ULX3 in its low-luminosity state), this candidate optical counterpart is on the bright end of the distribution (van Paradijs \& McClintock 1994), but again we cannot exclude the possibility that the donor is a lower mass star and the optical emission is dominated by an irradiated accretion disc.

Source 2 has absolute magnitudes and colours roughly consistent with those of red supergiants (potentially similar to NGC 300 ULX1, Heida et al. 2019). However, as this source displays variability in the F814W band, colours calculated from observations taken several years apart are obviously unreliable; multiband photometry taken at a single epoch, as well as a better determination of the local reddening, is necessary to determine the nature of this candidate counterpart. Detailed optical spectroscopy of these counterparts is not currently plausible, but may be possible with the next generation of 30-m class telescopes. 


\section{DISCUSSION AND CONCLUSIONS}

NGC 7090 ULX3 - aka 2CXO J213622.6 - 543234 and 4XMM $\mathrm{J} 213622.4$ - 543233 - is a newly discovered ULX in the galaxy NGC 7090, with a peak luminosity of $L_{\mathrm{X} \text {, peak }} \sim 6 \times 10^{39} \mathrm{erg} \mathrm{s}^{-1}$. This is the latest member of the growing population of transient ULXs (e.g. Soria et al. 2012; Middleton et al. 2012, 2013; Pintore et al. 2018, 2020; Earnshaw et al. 2019a, 2020; Brightman et al. 2020). Remarkably, ULX3 is the third such source in the galaxy NGC 7090 alone (e.g. Liu et al. 2019; Song et al. 2020).

Although we refer to ULX3 as a transient ULX, as it has only recently been seen to exhibit luminosities at the ULX level, it is not necessarily an X-ray transient in the more traditional sense. Most XRBs in our own Galaxy are transient LMXBs, which spend the majority of the time in quiescence $\left(L_{X} \sim 10^{30-34} \mathrm{erg} \mathrm{s}^{-1}\right.$; e.g. Homan et al. 2013; Reynolds et al. 2014), interspersed by transient outbursts of activity reaching much higher luminosities (which are widely expected to be related to the hydrogen ionization instability; Lasota 2001). Although a rare occurrence, some of these sources can reach peak luminosities similar to ULX3 (e.g. Middleton et al. 2012). However, ULX3 appeared to have a relatively stable luminosity of $\sim 10^{38} \mathrm{erg} \mathrm{s}^{-1}$ prior to its recent transition into the ULX regime. This potentially causes ULX3 to stand out from classic LMXBs, as such luminosities would very much be in the outburst regime for such sources. If either of the candidate optical counterparts seen in the HST observations are dominated by the donor star, ULX 3 could be accreting from a supergiant companion. However, the large increase in luminosity seen recently means ULX3 also stands out from classic wind-fed HMXBs, which tend to be (relatively) persistent. Some kind of Be/XRB-like phenomenon might be possible; in addition to the regular outbursts that occur when the compact object passes through the decretion disc that surrounds its stellar companion, these sources occasionally exhibit rare 'type II' outbursts which can reach superEddington luminosities and appear to be unrelated to the orbital dynamics of the system (see Reig 2011 for a review on Be/XRB systems). However, here too the apparently stable luminosity seen from ULX3 would be abnormally high. Unfortunately, given the sparse coverage, we can only place very loose constraints on the timescale over which ULX3 evolved into the ULX regime; given that the source was not detected in any of the individual Swift observations prior to our recent observing campaign, this must have occurred sometime between 2014 July and 2019 October, a window of $\sim 5 \mathrm{yr}$. However, this period of activity seems to have lasted $>7$ months, as ULX3 has been almost persistently detected by Swift over our 20192020 monitoring campaign; this would also be abnormally long for a type II outburst from a Be/XRB-like system.

The nature of the accretor in ULX3 is not clear from the current data. On the one hand, the spectral data are consistent with being dominated by emission from a standard thin disc with a constant inner radius for all observing epochs to date (implying in turn that the data are consistent with $L \propto T_{\text {in }}^{4}$, although we stress again that the uncertainties are large for the low-flux data). This may suggest the presence of a $\mathrm{BH}$ accretor. Taking these results at face value, we estimate a minimum value for the inner radius from the normalization of the DISKBB model for epoch XN1, ${ }^{4}$ as this is given by $\left[R_{\text {in }} /\left(D \xi f_{\text {col }}^{2}\right)\right]^{2} \cos (i)$. Here, $R_{\text {in }}$ and $D$ are in units of $\mathrm{km}$ and $10 \mathrm{kpc}$, respectively, while $f_{\text {col }}$ and $\xi$ are corrections that account for

\footnotetext{
${ }^{4}$ We also investigated a joint fit to all of the XMM-Newton, NUSTAR, and Chandra data (new and archival) with the DISKBB normalization linked across all epochs, but found that the results were no different to fitting epoch XN1 alone, as this dominates the total S/N.
}

the complex atmospheric physics in the disc and the fact that the peak temperature actually arises at a radius slightly larger than $R_{\text {in }}$, respectively. The product $\xi f_{\text {col }}^{2}$ is generally taken to be $\sim 1.2$ for a standard thin disc (Shimura \& Takahara 1995; Kubota et al. 1998). Taking $\cos (i)=1$ as a limiting case, we find $R_{\text {in }} \gtrsim 55 \mathrm{~km}$.

This is significantly larger than the standard neutron star radius $\left(R_{\mathrm{NS}} \sim 13 \mathrm{~km}\right.$, Riley et al. 2019; Miller et al. 2019) and assuming the disc reaches the innermost stable circular orbit of the accretor, would imply the presence of a BH with a minimum mass of $M_{\mathrm{BH}}>6-30 \mathrm{M}_{\odot}$ (for spin parameters $0 \leq a^{*} \leq 0.998$, i.e. $R_{\text {in }}=1-6 R_{\mathrm{G}}$, where $R_{\mathrm{G}}=$ $\left.G M_{\mathrm{BH}} / c^{2}\right)$. If we instead assume an intermediate inclination $(\cos (i)=$ 0.5 ) and an intermediate spin (such that $R_{\text {in }}=3 R_{\mathrm{G}}$ ), then this would imply $R_{\text {in }} \sim 75 \mathrm{~km}$ and in turn $M_{\mathrm{BH}} \sim 20 \mathrm{M}_{\odot}$. In addition, the thin nature of the best-fitting accretion disc model would imply that the luminosity never exceeds the source's Eddington limit, if also taken at face value. Although we have previously assumed isotropic emission, if the emission is truly dominated by a thin accretion disc then the inclination of the disc has to be accounted for when estimating the peak luminosity, and we find $L_{\mathrm{X} \text {, peak }} \sim 3 \times 10^{39} / \mathrm{cos}(i) \mathrm{erg} \mathrm{s}^{-1}$. For the average inclination expected for a randomly orientated disc, that is, $\cos (i)=0.5$, we return to $L_{\mathrm{X}}$, peak $\sim 6 \times 10^{39} \mathrm{erg} \mathrm{s}^{-1}$, which would in turn imply a lower limit to the $\mathrm{BH}$ mass of $M_{\mathrm{BH}} \gtrsim 40 \mathrm{M}_{\odot}$. This mass would be consistent with the DISKBB normalization for $a^{*}=0.998$. Interestingly, the disc temperature expected for a rapidly rotating $40 \mathrm{M}_{\odot} \mathrm{BH}$ accreting at close to its Eddington limit is $\sim 2 \mathrm{keV}$ (e.g. Makishima et al. 2000), similar to that observed from epoch XN1. Indeed, if we fit epoch XN1 with KERRBB, ${ }^{5}$ a fully relativistic thin disc model ( $\mathrm{Li}$ et al. 2005), instead of the simpler DISKBB model, fixing the spin to the maximal value, the inclination to $60^{\circ}$ and assuming a standard colour correction factor of $f_{\text {col }}=1.7$ (Shimura \& Takahara 1995; Davis \& El-Abd 2019), the best-fitting BH mass is $\sim 37 \mathrm{M}_{\odot}$.

Although such a BH would be larger than any seen in an XRB in our own Galaxy (e.g. Orosz 2003), similar mass BHs are known to exist as they are now fairly regularly being seen in $\mathrm{BH}-\mathrm{BH}$ mergers by LIGO (Abbott et al. 2019). The formation of such a $\mathrm{BH}$ via standard stellar evolution may require a low metallicity (e.g. Zampieri \& Roberts 2009; Belczynski et al. 2010). Although there is not much information regarding the metallicity of NGC 7090 available in the literature, we note that the majority of oxygen abundance estimates compiled by De Vis et al. (2019) would imply an abundance of $A_{\mathrm{O}} /$ solar $\sim 0.5$ (or alternatively $12+\log [\mathrm{O} / \mathrm{H}] \sim 8.4$ ). Smaller masses could still formally be permitted for other combinations of spin and inclination; we show the dependence of the best-fitting mass on the disc inclination for three spin parameters (non-rotating, moderately rotatin,g and maximally rotating) based on the KERRBB model in Fig. 7. However, this would push the peak luminosity into the super-Eddington regime, for which the KERRBB model is not formally valid. Furthermore, the colour correction factor used here may not be valid for accretion at/above the Eddington limit; higher values may be expected instead (e.g. Watarai \& Mineshige 2003; Kawaguchi 2003), in which case the mass estimates assuming $f_{\text {col }}=$ 1.7 would be underestimated by a factor of $\left(f_{\text {col }} / 1.7\right)^{2}$. Watarai \& Mineshige (2003) suggest that $f_{\text {col }} \sim 3$, in which case the best-fitting mass curves shown in Fig. 7 would systematically shift upwards by a factor of $\sim 3$.

On the other hand, the long-term flux distribution (although still fairly sparsely sampled in terms of sensitive observations) is consistent with ULX3 having a bi-modal flux distribution. This sort of distribution is broadly expected for neutron stars undergoing

\footnotetext{
${ }^{5}$ Note that we use the updated version described in Parker et al. (2019).
} 


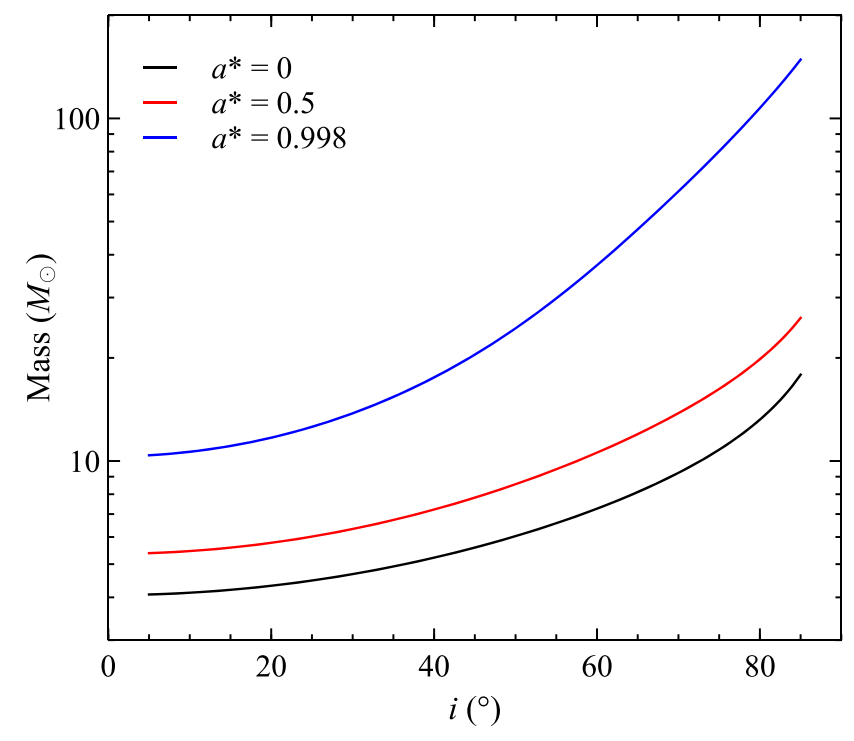

Figure 7. The dependence of the best-fitting mass from the KERRBB model (Li et al. 2005) on the inclination of the disc, assuming $f_{\text {col }}=1.7$, for three different spin parameters: non-rotating $\left(a^{*}=0\right)$, moderately rotating $\left(a^{*}=\right.$ $0.5)$, and maximally rotating $\left(a^{*}=0.998\right)$.

transitions to/from the propeller regime (Tsygankov et al. 2016), as the infalling material cannot pass through the magnetosphere $\left(R_{\mathrm{M}}\right)$ of the neutron star in the propeller regime, and so at some transition point the accretion rate (and thus the observed luminosity) drops precipitously. It may well be that flux distributions similar to that seen here are tentative evidence for a magnetic neutron star accretor. Many of the known ULX pulsars exhibit low-flux states (e.g. Motch et al. 2014; Walton et al. 2015a), and evidence for similar behaviour is now being seen in a growing number of sources among the broader ULX population (Earnshaw et al. 2018; Song et al. 2020). These events are potentially related to the propeller regime, requiring a magnetized neutron star accretor, although other possibilities certainly remain possible. Most notably, obscuration of the inner accretion flow by its outer regions/winds has also been invoked to explain high-amplitude variability events in some cases (e.g. Vasilopoulos et al. 2019), particularly where long-time-scale ( $\gtrsim 10$ s of days) super-orbital Xray periods with large variability amplitudes are present (Brightman et al. 2019, 2020; Vasilopoulos et al. 2020a; see also Middleton et al. 2015, 2018). While such an explanation would potentially need ULX3 to have an extremely long X-ray period, given the apparent stability of the low-flux state across many years prior to our recent observations, it is also interesting to note that the long-time-scale Xray periods in ULXs are themselves most robustly seen in the known ULX pulsar systems (e.g. Walton et al. 2016a; Hu et al. 2017; Fürst et al. 2018; Brightman et al. 2019, 2020; Vasilopoulos et al. 2020a). Although we do not have any significant detection of $\mathrm{X}$-ray pulsations from ULX3, the current limits are only mildly constraining, and the pulsations are seen to be transient in a number of the known ULX pulsars (e.g. Israel et al. 2017a; Sathyaprakash et al. 2019; Bachetti et al. 2020). Furthermore, as discussed earlier, the lack of spectral complexity similar to that seen in other known ULX pulsars could easily be purely due to a combination of relatively high absorption and low S/N at high energies. The latter in particular could easily prevent us from significantly detecting the emission from any central accretion columns.

In the magnetized neutron star scenario, the inner radius of the disc is set by the magnetospheric radius, rather than the radius of the neutron star itself. Standard accretion theory for magnetic neutron stars (i.e. assuming a dipolar field geometry and a thin accretion disc, the latter of which may not be formally appropriate here) implies that the magnetospheric radius is given by $R_{\mathrm{M}}=\left(3.9 \times 10^{8}\right) L_{37}^{-2 / 7} B_{12}^{4 / 7}$ for a $1.4 \mathrm{M}_{\odot}$ neutron star with a radius of $13 \mathrm{~km}$, where $L_{37}$ is the $\mathrm{X}$-ray luminosity in units of $10^{37} \mathrm{erg} \mathrm{s}^{-1}$ and $B_{12}$ is the magnetic field strength in units of $10^{12} \mathrm{G}$ (Lamb, Pethick \& Pines 1973; Cui 1997). The inner radius of $76 \mathrm{~km}$ estimated previously would therefore imply a rather weak field of $\sim 2 \times 10^{10} \mathrm{G}$. Typical field strengths for X-ray pulsars in our own Galaxy are $\sim 10^{12} \mathrm{G}$ (Caballero \& Wilms 2012), although it has previously been suggested that ULX pulsars specifically may have weak fields (e.g. Kluźniak \& Lasota 2015; King \& Lasota 2016). It is also worth noting, however, that stronger fields could still be accommodated for an equivalent $R_{\mathrm{M}}$ if the field geometry is higher order than the standard dipole assumed above, which is a possibility that has also been suggested for ULX pulsars (e.g. Israel et al. 2017a).

Ultimately, however, further observations that can provide improved constraints on the timing properties and/or the evolution of the accretion flow will be required to reveal the nature of ULX3.

\section{ACKNOWLEDGEMENTS}

The authors would like to thank the reviewer for their positive feedback, which helped to improve the final version of the manuscript. DJW and MJM acknowledge support from the Science and Technology Facilities Council (STFC) in the form of Ernest Rutherford Fellowships. PAE acknowledges UK Space Agency (UKSA) support. This research has made use of data obtained with NUSTAR, a project led by Caltech, funded by NASA and managed by the NASA Jet Propulsion Laboratory (JPL), and has utilized the NUSTARDAS software package, jointly developed by the Space Science Data Centre (SSDC; Italy) and Caltech (USA). This research has also made use of data obtained with $X M M-N e w t o n$, an ESA science mission with instruments and contributions directly funded by ESA Member States, as well as public data from the Swift data archive. Finally, this work has also made use of data obtained from the Chandra Source Catalog, provided by the Chandra X-ray Center (CXC) as part of the Chandra Data Archive, as well as data obtained from the 4XMM XMM-Newton serendipitous source catalogue, compiled by the 10 institutes of the XMM-Newton Survey Science Centre (SSC) selected by ESA.

\section{DATA AVAILABILITY}

All of the data underlying this article are either already publicly available from ESA's XMM-Newton Science Archive (https://www. cosmos.esa.int/web/xmm-newton/xsa), NASA's HEASARC archive (https://heasarc.gsfc.nasa.gov/), and NASA's Chandra Data Archive (https://cxc.harvard.edu/cda/), or will be from 2021 May.

\section{REFERENCES}

Abbott B. et al., 2019, Phys. Rev. X, 9, 031040

Abramowicz M. A., Czerny B., Lasota J. P., Szuszkiewicz E., 1988, ApJ, 332 , 646

Arnaud K. A., 1996, in Jacoby G. H., Barnes J., eds, ASP Conf. Ser., Vol. 101, Astronomical Data Analysis Software and Systems V. Astron. Soc. Pac., San Francisco, p. 17

Bachetti M., 2018, Astrophysics Source Code Library, record ascl:1805.019

Bachetti M. et al., 2013, ApJ, 778, 163

Bachetti M. et al., 2014, Nature, 514, 202 
Bachetti M. et al., 2020, ApJ, 891, 44

Bañados E. et al., 2018, Nature, 553, 473

Belczynski K., Bulik T., Fryer C. L., Ruiter A., Valsecchi F., Vink J. S., Hurley J. R., 2010, ApJ, 714, 1217

Brightman M. et al., 2016, ApJ, 816, 60

Brightman M. et al., 2018, Nat. Astron., 2, 312

Brightman M. et al., 2019, ApJ, 873, 115

Brightman M. et al., 2020, ApJ, 895, 127

Buccheri R. et al., 1983, A\&A, 128, 245

Caballero I., Wilms J., 2012, Mem. Soc. Astron. Ital., 83, 230

Carpano S., Haberl F., Maitra C., Vasilopoulos G., 2018, MNRAS, 476, L45

Cash W., 1979, ApJ, 228, 939

Chandra A. D., Roy J., Agrawal P. C., Choudhury M., 2020, MNRAS, 495, 2664

Cui W., 1997, ApJ, 482, L163

Davis S. W., El-Abd S., 2019, ApJ, 874, 23

De Vis P. et al., 2019, A\&A, 623, A5

Dolphin A. E., 2000, PASP, 112, 1383

Earnshaw H. P., Roberts T. P., Sathyaprakash R., 2018, MNRAS, 476, 4272

Earnshaw H. P. et al., 2019a, ApJ, 881, 38

Earnshaw H. P., Roberts T. P., Middleton M. J., Walton D. J., Mateos S., 2019b, MNRAS, 483, 5554

Earnshaw H. P. et al., 2020, ApJ, 891, 153

El Mellah I., Sundqvist J. O., Keppens R., 2019, A\&A, 622, L3

Evans P. A. et al., 2009, MNRAS, 397, 1177

Evans I. N. et al., 2020, in American Astronomical Society Meeting Abstracts. p. 154.05

Fürst F. et al., 2016, ApJ, 831, L14

Fürst F., Walton D. J., Stern D., Bachetti M., Barret D., Brightman M., Harrison F. A., Rana V., 2017, ApJ, 834, 77

Fürst F. et al., 2018, A\&A, 616, A186

Gaia Collaboration et al., 2018, A\&A, 616, A1

Garmire G. P., 'Bautz M. W., Ford P. G., Nousek J. A., Ricker G. R., Jr, 2003 , in Truemper J. E., Tananbaum H. D., eds, SPIE Conf. Ser., Vol. 4851, XRay and Gamma-Ray Telescopes and Instruments for Astronomy, SPIE, Bellingham, p. 28

Gehrels N. et al., 2004, ApJ, 611, 1005

Gladstone J. C., Roberts T. P., Done C., 2009, MNRAS, 397, 1836

Güver T., Özel F., 2009, MNRAS, 400, 2050

Harrison F. A. et al., 2013, ApJ, 770, 103

Heida M. et al., 2015, MNRAS, 453, 3511

Heida M., Jonker P. G., Torres M. A. P., Roberts T. P., Walton D. J., Moon D.-S., Stern D., Harrison F. A., 2016, MNRAS, 459, 771

Heida M. et al., 2019, ApJ, 883, L34

HI4PI Collaboration et al., 2016, A\&A, 594, A116

Homan J. et al., 2013, ApJ, 775, 9

Hu C.-P., Li K. L., Kong A. K. H., Ng C.-Y., Chun-Che Lin L., 2017, ApJ, 835, L9

Israel G. L. et al., 2017a, Science, 355, 817

Israel G. L. et al., 2017b, MNRAS, 466, L48

Jansen F. et al., 2001, A\&A, 365, L1

Kaaret P., Feng H., Roberts T. P., 2017, ARA\&A, 55, 303

Karachentsev I. D., Kaisina E. I., Makarov D. I., 2018, MNRAS, 479, 4136

Kawaguchi T., 2003, ApJ, 593, 69

King A., Lasota J.-P., 2016, MNRAS, 458, L10

King A. R., Pounds K. A., 2003, MNRAS, 345, 657

King A. R., Davies M. B., Ward M. J., Fabbiano G., Elvis M., 2001, ApJ, 552, L109

Kluźniak W., Lasota J.-P., 2015, MNRAS, 448, L43

Kosec P., Pinto C., Walton D. J., Fabian A. C., Bachetti M., Brightman M., Furst F., Grefenstette B. W., 2018, MNRAS, 479, 3978

Kubota A., Tanaka Y., Makishima K., Ueda Y., Dotani T., Inoue H., Yamaoka K., 1998, PASJ, 50, 667

Lamb F. K., Pethick C. J., Pines D., 1973, ApJ, 184, 271

Lasota J.-P., 2001, New Astron. Rev., 45, 449

Leahy D. A., Darbro W., Elsner R. F., Weisskopf M. C., Sutherland P. G., Kahn S., Grindlay J. E., 1983, ApJ, 266, 160

Lehmer B. D. et al., 2019, ApJS, 243, 3
Li L.-X., Zimmerman E. R., Narayan R., McClintock J. E., 2005, ApJS, 157, 335

Liu Z., O’Brien P. T., Osborne J. P., Evans P. A., Page K. L., 2019, MNRAS, 486, 5709

Madsen K. K. et al., 2015, ApJS, 220, 8

Madsen K. K., Grefenstette B. W., Pike S., Miyasaka H., Brightman M., Forster K., Harrison F. A., 2020, preprint (arXiv:2005.00569)

Makishima K., Kubota A., Mizuno T., Ohnishi T., Tashiro M., 2000, ApJ, 535,632

Martínez-Núñez S. et al., 2017, Space Sci. Rev., 212, 59

Middleton M. J., Sutton A. D., Roberts T. P., Jackson F. E., Done C., 2012, MNRAS, 420, 2969

Middleton M. J. et al., 2013, Nature, 493, 187

Middleton M. J., Heil L., Pintore F., Walton D. J., Roberts T. P., 2015, MNRAS, 447, 3243

Middleton M. J. et al., 2018, MNRAS, 475, 154

Miller M. C. et al., 2019, ApJ, 887, L24

Mineo S., Gilfanov M., Sunyaev R., 2012, MNRAS, 419, 2095

Mineshige S., Hirano A., Kitamoto S., Yamada T. T., Fukue J., 1994, ApJ, 426, 308

Misra D., Fragos T., Tauris T., Zapartas E., Aguilera-Dena D. R., 2020, A\&A, $642, \mathrm{~A} 174$

Mitsuda K. et al., 1984, PASJ, 36, 741

Mortlock D. J. et al., 2011, Nature, 474, 616

Motch C., Pakull M. W., Soria R., Grisé F., Pietrzyński G., 2014, Nature, 514, 198

Mukherjee E. S. et al., 2015, ApJ, 808, 64

Orosz J. A., 2003, in van der Hucht K., Herrero A., Esteban C., eds, IAU Symp., Vol. 212, A Massive Star Odyssey: From Main Sequence to Supernova. Astron. Soc. Pac., San Francisco, p. 365

Parker M. L., Buisson D. J. K., Tomsick J. A., Fabian A. C., Madsen K. K., Walton D. J., Furst F., 2019, MNRAS, 484, 1202

Pinto C., Middleton M. J., Fabian A. C., 2016, Nature, 533, 64

Pinto C. et al., 2017, MNRAS, 468, 2865

Pinto C. et al., 2020, MNRAS, 492, 4646

Pintore F. et al., 2018, MNRAS, 477, L90

Pintore F. et al., 2020, ApJ, 890, 166

Rana V. et al., 2015, ApJ, 799, 121

Ransom S. M., Eikenberry S. S., Middleditch J., 2002, AJ, 124, 1788

Reig P., 2011, Ap\&SS, 332, 1

Reynolds M. T., Reis R. C., Miller J. M., Cackett E. M., Degenaar N., 2014, MNRAS, 441, 3656

Riley T. E. et al., 2019, ApJ, 887, L21

Roberts T. P., Gladstone J. C., Goulding A. D., Swinbank A. M., Ward M. J., Goad M. R., Levan A. J., 2011, Astron. Nachr., 332, 398

Rodríguez Castillo G. A. et al., 2020, ApJ, 895, 60

Sathyaprakash R. et al., 2019, MNRAS, 488, L35

Schlafly E. F., Finkbeiner D. P., 2011, ApJ, 737, 103

Shakura N. I., Sunyaev R. A., 1973, A\&A, 24, 337

Shimura T., Takahara F., 1995, ApJ, 445, 780

Skinner G. K., Bedford D. K., Elsner R. F., Leahy D., Weisskopf M. C., Grindlay J., 1982, Nature, 297, 5867

Song X., Walton D. J., Lansbury G. B., Evans P. A., Fabian A. C., Earnshaw H., Roberts T. P., 2020, MNRAS, 491, 1260

Soria R., Kuntz K. D., Winkler P. F., Blair W. P., Long K. S., Plucinsky P. P., Whitmore B. C., 2012, ApJ, 750, 152

Steiner J. F., Narayan R., McClintock J. E., Ebisawa K., 2009, PASP, 121, 1279

Stobbart A.-M., Roberts T. P., Wilms J., 2006, MNRAS, 368, 397

Strüder L. et al., 2001, A\&A, 365, L18

Swartz D. A., Soria R., Tennant A. F., Yukita M., 2011, ApJ, 741, 49

Tao L., Kaaret P., Feng H., Grisé F., 2012, ApJ, 750, 110

Tao L. et al., 2019, ApJ, 873, 19

Tsygankov S. S., Mushtukov A. A., Suleimanov V. F., Poutanen J., 2016, MNRAS, 457, 1101

Turner M. J. L. et al., 2001, A\&A, 365, L27

van Haaften L. M., Maccarone T. J., Rhode K. L., Kundu A., Zepf S. E., 2019, MNRAS, 483, 3566 
van Paradijs J., McClintock J. E., 1994, A\&A, 290, 133

Vasilopoulos G., Petropoulou M., Koliopanos F., Ray P. S., Bailyn C. B., Haberl F., Gendreau K., 2019, MNRAS, 488, 5225

Vasilopoulos G., Lander S. K., Koliopanos F., Bailyn C. D., 2020a, MNRAS, 491, 4949

Vasilopoulos G. et al., 2020b, MNRAS, 494, 5350

Verbunt F., 1993, ARA\&A, 31, 93

Verner D. A., Ferland G. J., Korista K. T., Yakovlev D. G., 1996, ApJ, 465, 487

Walton D. J. et al., 2011a, MNRAS, 414, 1011

Walton D. J., Roberts T. P., Mateos S., Heard V., 2011 b, MNRAS, 416, 1844

Walton D. J. et al., 2014, ApJ, 793, 21

Walton D. J. et al., 2015a, ApJ, 799, 122

Walton D. J. et al., 2015b, ApJ, 806, 65

Walton D. J. et al., 2016a, ApJ, 827, L13

Walton D. J. et al., 2016b, ApJ, 826, L26
Walton D. J. et al., 2016c, ApJ, 826, 87

Walton D. J. et al., 2017, ApJ, 839, 105

Walton D. J. et al., 2018a, ApJ, 857, L3

Walton D. J. et al., 2018b, MNRAS, 473, 4360

Walton D. J. et al., 2018c, ApJ, 856, 128

Walton D. J. et al., 2020, MNRAS, 494, 6012

Watarai K.-y., Mineshige S., 2003, ApJ, 596, 421

Webb N. A. et al., 2020, A\&A, 641, A136

Weisskopf M. C., Brinkman B., Canizares C., Garmire G., Murray S., Van Speybroeck L. P., 2002, PASP, 114, 1

Wilms J., Allen A., McCray R., 2000, ApJ, 542, 914

Wilson-Hodge C. A. et al., 2018, ApJ, 863, 9

Zampieri L., Roberts T. P., 2009, MNRAS, 400, 677

This paper has been typeset from a $\mathrm{T}_{\mathrm{E}} \mathrm{X} / \mathrm{LT}_{\mathrm{E}} \mathrm{X}$ file prepared by the author. 